Table 1. Characteristics of SLE patients enrolled in this study

\begin{tabular}{lc}
\hline Characteristics & $\mathrm{n}=54, \mathrm{no} .(\%)$ \\
\hline Female, no(\%) & $51(94)$ \\
Age, years, mean \pm SD & $41.9 \pm 12.8$ \\
Disease duration, years, mean \pm SD & $15.1 \pm 11.1$ \\
Past involvement & \\
Renal involvement & $23(43)$ \\
NPSLE & $5(9)$ \\
Complication & $10(19)$ \\
APS & $2(4)$ \\
Dyslipidemia & $1(2)$ \\
Diabetes & \\
Concomitant immunosuppressive treatments & \\
Prednisone & $46(85)$ \\
$\quad$ No.(\%) & $5.0(1-10)$ \\
Median Dosage, mg/day (range) & $3.9 \pm 2.0$ \\
Disease activity & $30(56)$ \\
SLENA-SLEDAl score & $21(39)$ \\
$\quad$ Current skin involvement & $29(54)$ \\
low compli-dsDNA positive, no(\%) & \\
\hline
\end{tabular}

Anti-dsDNA positive means anti ds-DNA titer increases over $12 \mathrm{IU} / \mathrm{mlLow}$ complement means any of $\mathrm{C} 3, \mathrm{C} 4$ and $\mathrm{CH} 50$ decreases to less $68 \mathrm{mg} / \mathrm{dl}$, less $12 \mathrm{mg} / \mathrm{dl}, 30 \mathrm{U} / \mathrm{ml}$.APS: Antiphospholipid antibody syndrome, NPSLE: neuropsychiatric SLE,

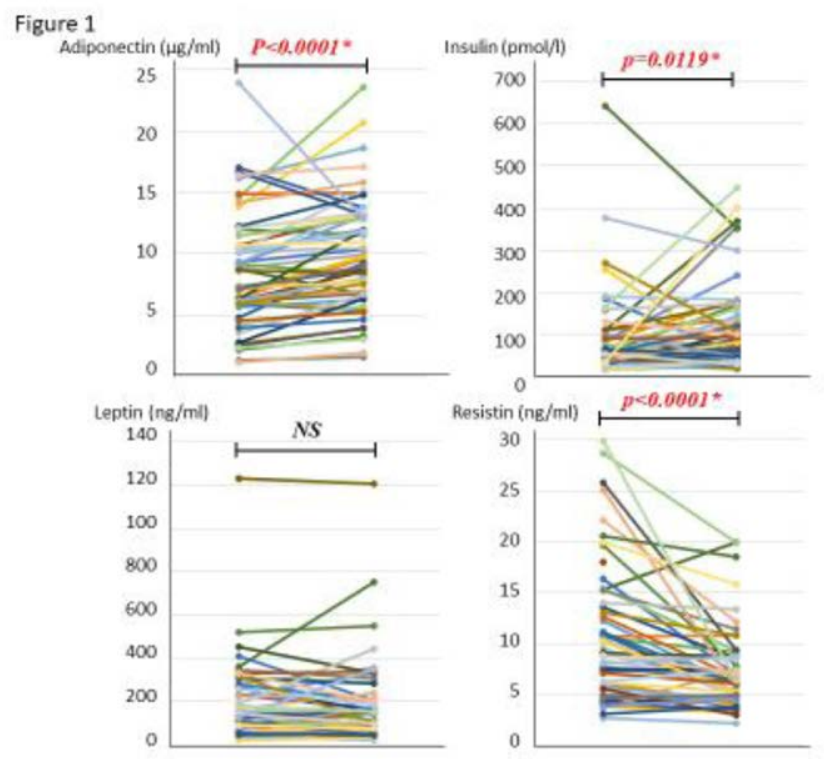

Disclosure of Interests: None declared

DOI: 10.1136/annrheumdis-2021-eular.2362

\section{AB0288 2 SAFETY OF BELIMUMAB IN PATIENTS WITH ACTIVE SYSTEMIC LUPUS ERYTHEMATOSUS: YEAR 2 FOLLOW-UP OF A LARGE PHASE 4, RANDOMISED, DOUBLE-BLIND, PLACEBO-CONTROLLED STUDY}

S. Sheikh ${ }^{1,2}$, M. Scheinberg ${ }^{3}$, J. C. C. Wei ${ }^{4,5}$, D. Tegzová6 ${ }^{6}$, W. Stohl ${ }^{7}$, T. Mucenic $^{8}$, R. Punwaney ${ }^{9}$, R. Kurrasch ${ }^{10}$, J. Harris ${ }^{11}$, S. Muzaffar ${ }^{12}$, S. Fernandes ${ }^{13}$, N. L. Fox ${ }^{9}$, A. Liu ${ }^{14}$, H. Quasny ${ }^{15}$, D. Roth ${ }^{16} .{ }^{1}$ University of North Carolina, Thurston Arthritis Research Center, Chapel Hill, NC, United States of America; ${ }^{2}$ University of North Carolina at Chapel Hill School of Medicine, Department of Medicine, Division of Rheumatology, Allergy and Immunology, Chapel Hill, NC, United States of America; ${ }^{3}$ Hospital Abreu Sodré, Centro de Pesquisas Clinicas, Sao Paulo, Brazil; ${ }^{4}$ Institute of Medicine, Chung Shan Medical University, Taichung, Taiwan, Republic of China; ${ }^{5}$ Graduate Institute of Integrated Medicine, China Medical University, Taichung, Taiwan, Republic of China; Institute of Rheumatology, Department of Rheumatology, Prague, Czech Republic; ${ }^{7}$ University of Southern California Keck School of Medicine, Division of Rheumatology, Los Angeles, CA, United States of America; ${ }^{8}$ Moinhos de Vento, Moinhos de Vento, Porto Alegre, Brazil; ${ }^{9}$ GlaxoSmithKline, Clinical Development, Collegeville, PA, United States of America; ${ }^{10} \mathrm{GlaxoSmithKline,}$ Immunoinflammation, Collegeville, PA, United States of America;

${ }^{11}$ GlaxoSmithKline, Statistics, Uxbridge, United Kingdom; ${ }^{12}$ GlaxoSmithKline, Clinical Science, Stevenage, United Kingdom; ${ }^{13}$ GlaxoSmithKline, Medical Affairs, Stevenage, United Kingdom; ${ }^{14}$ GlaxoSmithKline, Global Clinical Safety and Pharmacovigilance, Uxbridge, United Kingdom; ${ }^{15}$ GlaxoSmithKline, Immunology \& Specialty Medicine, Chapel Hill, NC, United States of America;

${ }^{16}$ GlaxoSmithKline, Research and Development, Collegeville, PA, United States of America

Background: Belimumab (BEL), a recombinant human monoclonal antibody that inhibits B-lymphocyte stimulator (BLyS), is approved for the treatment of systemic lupus erythematosus (SLE). Clinical studies have yielded varying incidence rates of mortality and adverse events of special interest, such as malignancies, thereby necessitating large-scale, long-term assessment following BEL exposure.

Objectives: To assess all-cause mortality and new primary malignancies during post-treatment Year 2 follow-up in adult patients with active, autoantibody-positive SLE who received intravenous (IV) BEL or placebo (PBO), plus standard therapy in the 52-week double-blind treatment period of the ongoing BASE trial. Methods: This was a post-treatment follow-up of the Phase 4, double-blind study (BASE ${ }^{1}$; GSK Study BEL115467; NCT01705977), which randomised 4019 adults with active SLE and receiving standard therapy to BEL (10 mg/kg IV) or PBO on Days $0,14,28$, and monthly thereafter until Week 48. All patients (including those who discontinued BEL before the end-of-treatment phase) were contacted by phone annually (+/-30-day time window). Rates of mortality and new primary malignancy are summarised for Year 2 follow-up, presented by the treatment received during the 52-week double-blind treatment period (Year 1).

Results: Baseline patient characteristics and disease activity collected at the start of the study, evaluated in patients with Year 2 follow-up were similar to the overall Year 1 study population. Cumulatively by Year 2 follow-up, 10.7\% and $9.5 \%$ of patients had been exposed to commercial BEL in the BEL and PBO groups, respectively. Cumulative follow-up adjusted mortality and malignancy rates (per 100 patient years) were lower in the BEL vs PBO Year 1 treatment group (Table 1)

Conclusion: Year 2 follow-up results of BASE, the largest clinical trial of SLE to date, ${ }^{1}$ provide continued support for the BEL safety profile. No new BEL safety concerns were identified in patients with active, autoantibody-positive SLE receiving standard therapy.

Funding: GSK

REFERENCES:

[1] Sheikh SZ, et al. Lancet Rheum. 2020 (ePub ahead of print) doi.org/10.1016/ S2665-9913(20)30355-6

Table 1. Year 2 post-treatment* follow-up mortality and new primary malignancy rates by study treatment during Year 1

\begin{tabular}{lccc}
\hline & BEL & PBO & Total \\
\hline Year 1 as-treated population & $\mathrm{N}=\mathbf{2 0 0 2}$ & $\mathrm{N}=\mathbf{2 0 0 1}$ & $\mathrm{N}=\mathbf{4 0 0 3}$ \\
\hline Year 1 deaths, n (\%) & $13(0.65)$ & $22(1.10)$ & $35(0.87)$ \\
Year 1 new primary malignancies, $\mathrm{n}(\%)$ & $9(0.45)$ & $10(0.50)$ & $19(0.47)$ \\
Year 2 (as-treated in Year 1) population & $\mathrm{N}=1681$ & $\mathrm{~N}=1666$ & $\mathrm{~N}=3347$ \\
Year 2 deaths by MedDRA SOC, $\mathbf{n}(\%)$ & $9(0.54)$ & $21(1.26)$ & $30(0.90)$ \\
Cardiac disorders & $2(0.12)$ & $6(0.36)$ & $8(0.24)$ \\
Infections and infestations & $4(0.24)$ & $2(0.12)$ & $6(0.18)$ \\
Uncoded & $1(0.06)$ & $3(0.18)$ & $4(0.12)$ \\
General disorders/administration site conditions & $1(0.06)$ & $2(0.12)$ & $3(0.09)$ \\
Gastrointestinal disorders & $1(0.06)$ & $1(0.06)$ & $2(0.06)$ \\
Neoplasms & 0 & $2(0.12)$ & $2(0.06)$ \\
Other & 0 & $5(0.30)$ & $5(0.15)$ \\
Cumulative deaths by Year 2 follow-up, $\mathbf{n}$ (\%) & $22(1.10)$ & $43(2.15)$ & $65(1.62)$ \\
Incidence rate per 100 patient years & 0.60 & 1.18 & 0.89 \\
Year 2 new primary malignancies by MedDRA SOC, $\mathbf{n}(\%)$ & $3(0.18)$ & $4(0.24)$ & $7(0.21)$ \\
Neoplasms & $2(0.12)$ & $4(0.24)$ & $6(0.18)$ \\
Hepatobiliary disorders & $1(0.06)$ & 0 & $1(0.03)$ \\
Cumulative malignancies by Year 2 follow-up, $\mathbf{n}(\%)$ & $12(0.60)$ & $14(0.70)$ & $26(0.65)$ \\
Patient incidence rate per 100 patient years & 0.34 & 0.40 & 0.37 \\
\hline
\end{tabular}

${ }^{*}$ Patients in the post-treatment follow-up period are no longer receiving study treatment; ${ }^{\dagger}$ event/patient: blood/lymphatic system, musculoskeletal/connective tissue, nervous system, psychiatric, and renal/urinary disorders.MedDRA, Medical Dictionary for Regulatory Activities $\mathrm{SOC}$, system organ class.

Acknowledgements: Medical writing assistance was provided by Katalin Bartus, PhD, Fishawack Indicia Ltd., UK, part of Fishawack Health, and was funded by GSK.

Disclosure of Interests: Saira Sheikh Grant/research support from: Pfizer, Morton Scheinberg Consultant of: GSK, Pfizer, Alnylam, AbbVie, PTC Therapeutics, James Cheng-Chung Wei Consultant of: TSH Biopharm, AbbVie, BMS Celgene, Chugai, Eisai, Janssen, Novartis, Pfizer, Sanofi-Aventis and UCB pharma, Grant/research support from: AbbVie, Amgen, Astellas, BMS, Celgene, Eli Lilly, Gilead, Janssen, Novartis, Pfizer Sun and UCB, Dana Tegzová: None declared, William Stohl Consultant of: GSK, Grant/research support from: GSK, Pfizer, Gilead, Tamara Mucenic Speakers bureau: Novartis, Janssen, BMS, AbbVie, Pfizer, Roche, Grant/research support from: GSK, Janssen, Roche, Eli Lilly, 
Gilead, UCB, Raj Punwaney Shareholder of: GSK, Employee of: GSK, Regina Kurrasch Shareholder of: GSK, Employee of: GSK, Julia Harris Shareholder of: GSK, Employee of: GSK, Saima Muzaffar Shareholder of: GSK, Employee of: GSK, Sofia Fernandes Shareholder of: GSK, Employee of: GSK, Norma Lynn Fox Shareholder of: GSK, Employee of: GSK, Andrew Liu Shareholder of: GSK, Employee of: GSK, Holly Quasny Shareholder of: GSK, Employee of: GSK, David Roth Shareholder of: GSK, Employee of: GSK DOI: 10.1136/annrheumdis-2021-eular.2552

\section{AB0289 COMPARED TO CLINICIAN RECORDED BILAG- 2004 MUSCULOSKELETAL SYSTEM SCORES - DISCORDANCE BETWEEN PATIENTS AND CLINICIANS}

E. Olech ${ }^{1,2}$, D. Stull ${ }^{3}$, B. Williams ${ }^{3}$, S. Bean ${ }^{3}$, G. Abreu ${ }^{4}$, E. Schwetje $^{5}$ R. Tummala ${ }^{5}$, S. O'quinn $6 .{ }^{1} I Q V I A$, Rheumatology Center of Excellence Therapeutic Strategy \& Science Unit, San Diego, United States of America; ${ }^{2} I Q V I A$, Rheumatology Center of Excellence, Las Vegas, United States of America; ${ }^{3} I Q V I A$, Patient Centered Endpoints, New York, United States of America; ${ }^{4}$ AstraZeneca, BioPharmaceuticals R\&D, Gothenburg, Sweden; ${ }^{5}$ AstraZeneca, BioPharmaceuticals R\&D, Gaithersburg, United States of America; ${ }^{6}$ AstraZeneca, BioPharmaceuticals Medical: Evidence, Gaithersburg, United States of America

Background: The musculoskeletal organ system BILAG-2004 (MSK BILAG) assessment is of critical importance in SLE clinical trials. Severe active polyarthritis, MSK BILAG A, by definition includes significant impairment of basic activities of daily living (ADLs), as opposed to MSK BILAG C, D, or E where ability to perform ADLs is expected to be preserved. In clinical trials, BILAG is scored by clinicians without formal review of patient reported outcomes (PROs). The Physical Health domain of the LupusQoL (LQol PH) (range $0-100$ ) can be used to assess the patient's physical function and ADLs. LQoL PH score thresholds defining impairment severity have not been established; however, a transformed LQoL PH score $\leq 50$ suggests more impaired function, which would not be expected in MSK BILAG C, D, or E. Conversely, a score $>50$ implies no major issues with ADLs, which would be contradictory to the definition of MSK BILAG A.

Objectives: To assess correlation of patient reported LQLL PH with MSK BILAG scores recorded by clinicians at various timepoints using data from the phase 3 TULIP studies ${ }^{1,2}$ and to investigate the percent of discordance between patients and clinicians.

Methods: Data from TULIP 1 and 2 studies (anifrolumab $300 \mathrm{mg}$ and placebo arms) were pooled to evaluate the relationship between LQoL PH and MSK BILAG scores at baseline, weeks 24 and 52 using Spearman correlations as post-hoc analysis. Mean LQoL PH scores were assessed for each MSK BILAG category at the three timepoints using one-way ANOVA. Percent of patients with MSK BILAG A and LQoL PH scores $>50$ and patients with MSK BILAG C, D, or $E$ and LQoL PH scores $\leq 50$ was calculated at baseline, week 24 and 52. MSK BILAG B was excluded from the analysis because discordance could not be easily defined for this category compared with the more extreme MSK BILAG categories.

Results: Total of 690 patients were included in the pooled analysis (Table 1). Significant correlations between LQoL PH and MSK BILAG scores were found at each time point (nominal $p<0.0001$ ); this relationship became stronger over time. Mean LQLL PH scores were different in each MSK BILAG category, with the highest in MSK BILAG D/E and the lowest in the MSK BILAG A category, thus confirming the discriminatory ability of the LQoL PH (Table 1).

Table 1. Correlation coefficients (CC) between LQoL PH and MSK BILAG scores, and mean LQoL PH scores with standard deviations (SD) per each MSK BILAG category at baseline, weeks 24 and 52 .

\begin{tabular}{lcccccc}
\hline & Baseline & \multicolumn{2}{c}{ Week 24 } & \multicolumn{2}{c}{ Week 52 } \\
\hline & CC & $\mathrm{N}$ & CC & $\mathrm{N}$ & CC & $\mathrm{N}$ \\
\hline Total Population & -0.25 & 690 & $\begin{array}{c}-0.36 \\
\text { Mean LQL PH }\end{array}$ & 626 & $\begin{array}{c}-0.41 \\
\text { Mean LQL PH }\end{array}$ & 552 \\
MSK BILAG & $\begin{array}{c}\text { Mean LQL PH } \\
\text { Score (SD) }\end{array}$ & & Score (SD) & & Score (SD) & \\
$0(\mathrm{D} / \mathrm{E})$ & $69.3(24.7)$ & 17 & $74.2(22.1)$ & 186 & $74.5(21.3)$ & 237 \\
$1(\mathrm{C})$ & $62.3(25.4)$ & 60 & $64.0(23.9)$ & 233 & $60.6(22.5)$ & 184 \\
$8(\mathrm{~B})$ & $56.6(24.4)$ & 398 & $55.1(24.2)$ & 163 & $51.3(24.3)$ & 105 \\
$12(\mathrm{~A})$ & $44.9(25.8)$ & 215 & $43.9(25.9)$ & 44 & $44.2(26.2)$ & 26 \\
\hline
\end{tabular}

At baseline, $40 \%$ of patients who were assessed by clinicians as having MSK BILAG A reported minimal impairment in physical function and ADLs (LQoL PH $>50$ ) and $24.1 \%$ who had MSK BILAG C, D, or E reported difficulties with ADLs
(LQoL HP $\leq 50$ ), suggesting discordance between patients and clinicians. This discordance slightly decreased over time (Figure 1).

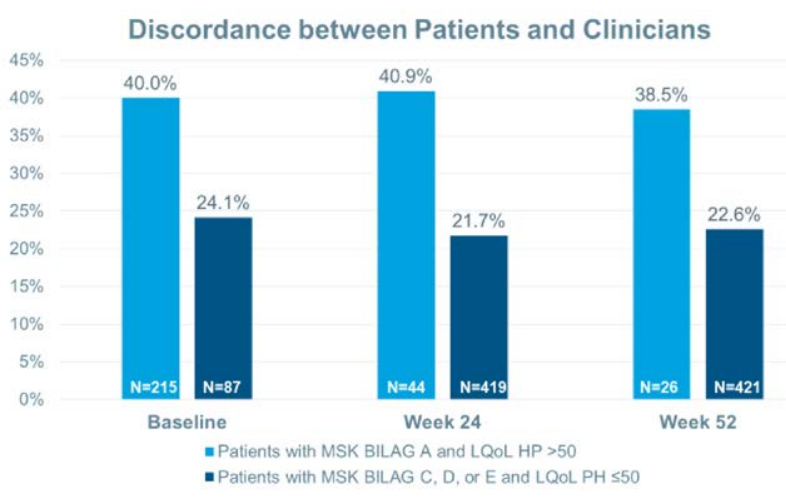

Figure 1. Percent of patients with MSK BILAG A and LQoL PH scores $>50$ and patients with MSK BILAG C, D, or E and LQoL PH scores $\leq 50$ at baseline, weeks 24 and 52 .

Conclusion: Patient reported LQLL PH scores correlated with MSK BILAG scores and showed discriminant validity for MSK BILAG scores. Greater discordance was seen between LQoL PH and MSK BILAG A compared with C, D, or $\mathrm{E}$. These findings suggest a need for further investigation of a role for PROs in MSK BILAG scoring. Formal review of PROs by clinicians during MSK BILAG assessment could be considered in future SLE clinical trials.

\section{REFERENCES:}

[1] Furie $\mathrm{R}$ et al. Lancet 2019

[2] Morand EF et al. N Engl J Med 2020

Acknowledgements: This study was sponsored by AstraZeneca.

Disclosure of Interests: Ewa Olech Speakers bureau: Abbvie, Amgen, Merck Pfizer, and UCB, Grant/research support from: BMS, Donald Stull: None declared, Betsy Williams: None declared, Stephanie Bean: None declared, Gabriel Abreu Employee of: AstraZeneca, Erik Schwetje Employee of: AstraZeneca, Raj Tummala Employee of: AstraZeneca, Sean O'Quinn Shareholder of: AstraZeneca, Employee of: AstraZeneca

DOI: 10.1136/annrheumdis-2021-eular.2560

\section{AB0290 \\ TREATMENT OF INTERSTITIAL LUNG INVOLVEMENT IN SYSTEMIC LUPUS ERYTHEMATOSUS: EXPERIENCE IN A THIRD LEVEL HOSPITAL}

1. González Fernández ${ }^{1}$, C. Moriano ${ }^{1}$, E. Diez Álvarez ${ }^{1}$, C. Sieiro Santos ${ }^{1}$, C. Álvarez Castro ${ }^{1}$, M. Martín Martín ${ }^{1}$, M. E. Vallejo Pascual ${ }^{2}$, M. T. Perez Sandoval $1 .{ }^{1}$ Complejo Asistencial Universitario de León, Reumatología, León, Spain; ${ }^{2}$ Universidad de León, Facultad de CC. EE y Empresariales, León, Spain

Background: There are few studies ${ }^{1}$ on the treatment of diffuse interstitial lung disease (ILD) associated with systemic lupus erythematosus (SLE). Corticosteroids are usually used in cases of mild to moderate decrease in diffusion and the association with immunosuppressants (Cyclophosphamide, Rituximab...) is reserved for cases of more severe affectation.

Objectives: Assess the main treatments used in ILD associated with SLE in a third level hospital and analyze the therapeutic response through improvement in imaging tests and in respiratory function studies.

Methods: Descriptive observational study of patients diagnosed with SLE (SLICC criteria) who developed ILD in our hospital between 1973 and 2020. As variables of response to treatment, HRCT pattern at diagnosis and at two years, respiratory function tests at diagnosis and at one year were analyzed. Likewise, the different treatments used before and after diagnosis were studied such as hydroxychloroquine (HCQ), prednisone, methotrexate (MTX), Rituximab (RTX), belimumab (BLM), Cyclophosphamide (CFM), azathioprine (AZA) and mycophenolate mofetil (MMF).

Results: A total of 455 patients diagnosed with SLE were included, of which 20 had ILD (4.4\%). $65 \%$ of the analyzed sample were women with a mean age at diagnosis of $63 \pm 16.23$ years. $30 \%$ presented ILD as the first clinical data for the diagnosis of SLE. Only 3 patients died (due to causes other than ILD and SLE). At diagnosis, $90 \%$ had symptoms such as dyspnea (75\%) and cough $(60 \%)$.

$85 \%$ had alterations in the X-ray and $100 \%$ in the HRCT, where the predominant pattern was NINE (65\%). Spirometry showed an obstructive (25\%), restrictive $(20 \%)$ and normal $(55 \%)$ pattern; $90 \%$ had decreased diffusion, the majority being mild $(67 \%)$. With the treatment administered, HRCT stability was observed 